WellBeing International

WBI Studies Repository

5-15-2016

\title{
Laterality strength is linked to stress reactivity in Port Jackson sharks (Heterodontus portusjacksoni)
}

\author{
Evan E. Byrnes \\ Macquarie University \\ Catarina Vila Pouca \\ Macquarie University \\ Culum Brown \\ Macquarie University
}

Follow this and additional works at: https://www.wellbeingintlstudiesrepository.org/latera

Part of the Animal Studies Commons, Comparative Psychology Commons, and the Other Animal Sciences Commons

\section{Recommended Citation}

Byrnes, E. E., Pouca, C. V., \& Brown, C. (2016). Laterality strength is linked to stress reactivity in Port Jackson sharks (Heterodontus portusjacksoni). Behavioural brain research, 305, 239-246. https://doi.org/ 10.1016/j.bbr.2016.02.033

This material is brought to you for free and open access by WellBeing International. It has been accepted for inclusion by an authorized administrator of the WBI Studies Repository. For more information, please contact wbisr-info@wellbeingintl.org.

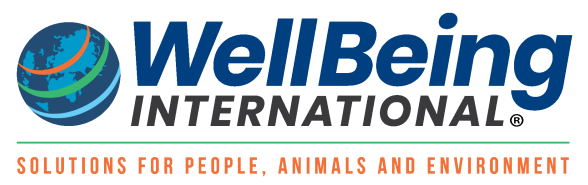




\section{Accepted Manuscript}

Title: Laterality strength is linked to stress reactivity in Port Jackson sharks (Heterodontus portusjacksoni)

Author: Evan E. Byrnes Catarina Vila-Pouca Culum Brown

PII: $\quad$ S0166-4328(16)30110-3

DOI: $\quad$ http://dx.doi.org/doi:10.1016/j.bbr.2016.02.033

Reference: $\quad$ BBR 10069

To appear in: $\quad$ Behavioural Brain Research

Received date: $\quad 9-10-2015$

Revised date: $\quad 20-2-2016$

Accepted date: $\quad$ 26-2-2016

Please cite this article as: Byrnes Evan E, Vila-Pouca Catarina, Brown Culum.Laterality strength is linked to stress reactivity in Port Jackson sharks (Heterodontus portusjacksoni).Behavioural Brain Research http://dx.doi.org/10.1016/j.bbr.2016.02.033

This is a PDF file of an unedited manuscript that has been accepted for publication. As a service to our customers we are providing this early version of the manuscript. The manuscript will undergo copyediting, typesetting, and review of the resulting proof before it is published in its final form. Please note that during the production process errors may be discovered which could affect the content, and all legal disclaimers that apply to the journal pertain. 
Laterality strength is linked to stress reactivity in Port Jackson sharks

(Heterodontus portusjacksoni)

Evan E. Byrnes ${ }^{\mathrm{a} 1}$, Catarina Vila-Pouca ${ }^{\mathrm{a}}$, Culum Brown ${ }^{\mathrm{a}}$

aDepartment of Biological Sciences, Macquarie University, North Ryde, NSW 2109

Australia

\footnotetext{
${ }^{1}$ Author to whom correspondence should be addressed. Tel: +610432761233 , email: evan.byrnes@students.mq.edu.au, 2a Edgecliff Esplanade, Seaforth, NSW 2092, Australia
} 


\title{
Highlights
}

- Lateralization in Heterodontus portusjacksoni appeared to be highly individualized

- Stronger lateralized individuals were more reactive to stress

- Laterality did not correlate with boldness in H. portusjacksoni

- Female H. portusjacksoni were more lateralized than males

\begin{abstract}
Cerebral lateralization is an evolutionarily deep-rooted trait, ubiquitous among the vertebrates and present even in some invertebrates. Despite the advantages of cerebral lateralization in enhancing cognition and facilitating greater social cohesion, large within population laterality variation exists in many animal species. It is proposed that this variation is maintained due links with inter-individual personality trait differences. Here we explored for lateralization in Port Jackson sharks (Heterodontus portusjacksoni) using T-maze turn and rotational swimming tasks. Additionally, we explored for a link between personality traits, boldness and stress reactivity, and cerebral lateralization. Sharks demonstrated large individual and sex biased laterality variation, with females demonstrating greater lateralization than males overall. Stress reactivity, but not boldness, was found to significantly correlate with lateralization strength. Stronger lateralized individuals were more reactive to stress. Demonstrating laterality in elasmobranchs for the first time indicates ancient evolutionary roots of vertebrate lateralization approximately 240 million years old. Greater lateralization in female elasmobranchs may be related enhancing females' ability to process multiple stimuli during mating, which could increase survivability and facilitate insemination. Despite contrasting evidence in teleost fishes, the results of this study suggest that stress reactivity, and other personality traits, may be linked to variation in lateralization.
\end{abstract}

Keywords: lateralization; stress reactivity; boldness; coping style; personality;

\author{
elasmobranch
}




\section{Introduction}

Over the past three decades the demonstration of lateralization amongst all vertebrate groups and some invertebrates suggests that it is an evolutionarily deep-rooted trait and may even predate its evolution in vertebrates [1-3]. Much of recent evolutionary research has focused on the advantages and disadvantages of lateralization to understand the selective mechanisms for the evolution of cerebral lateralization, such as the enhancement of cognitive functions. For example, more strongly lateralized individuals are quicker to learn tasks and can process greater cognitive loading [4, 5]. Additionally, lateralization enhances cohesive behaviours, such as shoaling and group foraging [6]. In visually complex habitats, such as coral reefs, the enhanced ability to process multiple stimuli and cooperate has been shown to enhance survivorship when predator abundance is high $[7,8]$.

Despite these apparent fitness advantages of cerebral lateralization, substantial individual variation in both direction and strength of lateralization exists in many species [9-12]. The underlying mechanisms maintaining this individual variation are still poorly understood, but a recent hypothesis proposes that variation in lateralization is maintained through a link with consistent inter-individual personality and behavioural differences [13]. Aggression, for example, appears to be a highly lateralized trait among vertebrates and invertebrates $[1,14,15]$. During antagonistic responses, animals direct aggressive displays to conspecifics on the left side more than on the right side. This asymmetry has been recorded in toads [16], lizards [17], domestic chicks [18], gelada baboons [19], horses [20], and fruit flies [21]. On the other hand, other personality traits reveal confounding relationships that vary by sex and/or species [22]. Irving and Brown [23] 
found little evidence that laterality relates to boldness, sociability, or activity in Poecilia reticulata, and populations of the poeciliid Brachyraphis episcopi vary in strength of lateralization [9, 24], as well as boldness [25], aggression [26], exploration, and activity [27].

The bulk of research that has examined the personality/lateralization relationship has focused on aggression and boldness; however, there is a paucity of research that has focused on stress reactivity (aka coping style or emotional reactivity). The few existing studies indicated that stress reactivity correlates with aggression, and suggested the existence of a relationship between stress reactivity and laterality [28]. Additionally, researchers commonly take advantage of the fact that laterality biases tend to be more pronounced in stressful situations, such as presence of predators or potential competitors. For example, when Bisazza \& Vallortigara [29] placed male mosquitofish (Gambusia holbrooki) placed in a circular tank, laterality biases were only apparent when a predator was present in the middle of the tank; not during spontaneous swimming or when a female was used as a stimulus.

Measurement of physiological parameters have demonstrated that the right cerebral hemisphere is associated with expression of intense, often negative, emotions and controls hormonal states that accompany these states [30, 31]. Therefore, it is expected that more strongly right lateralized individuals would exhibit stronger emotional and stress responses. Recent evidence that right lateralized marmosets were more pessimistic and avoided potentially stressful stimuli more than left lateralized conspecifics corroborate this prediction [32]. However, studies in dogs and domesticated chicks have shown that nonlateralized individuals to be more reactive, potentially as a 
result of the negative relationship between the development of lateralization and corticosterone levels during embryonic development $[33,34]$. While the relationship remains equivocal at best, it is apparent that studying the relationship between stress reactivity and laterality may provide valuable insight into the maintenance of individual variation in lateralization.

Studying lateralization in fishes has presented an especially interesting system for examining the evolution of vertebrate lateralization [15]. Fishes occur in a wide range of habitats that provides an opportunity to comparatively study the ecological conditions under which lateralization likely first evolved [1,35]. Additionally, in many fishes, optic fields do not overlap and primary visual projections ascend to neural structures on the contralateral hemisphere of the brain. Hence, observation of asymmetries is made particularly simple by examining which eye individuals use to observe particular cues or scenes $[10,29]$. Lateralized behaviours in fishes include inspection of conspecifics and predators [10, 24], initiation of aggressive interactions [36], shoaling tendencies [6], predatory approaches [37], exploration [9, 38], spatial abilities [5, 39], and communication [40]. While teleost fishes have received a bulk of attention from research examining the evolutionary origins of lateralization in vertebrates, no study has yet examined laterality in elasmobranchs - one of the oldest vertebrate lineages, appearing some 450 million years ago.

Here we examined behavioural lateralization in Port Jackson sharks (Heterodontus portusjacksoni), a species thought to originate approximately 240 million years ago [41], using two different lateralization tests. We employed turn preferences in a T-maze and directional preferences for rotational swimming, two tests that have been 
successfully used to determine laterality in teleost fishes. Given the relationship between laterality and social interactions in teleost fishes and the social aggregating behaviours of Port Jackson sharks [42, 43], we hypothesized that Port Jackson sharks will demonstrate a highly lateralized population bias, but we cannot predict the direction of laterality. We then examined for consistency of lateralization between turn and rotational preference. Lastly, sharks ' boldness and stress reactivity were scored in a previous experiment [44], and relationships between these traits and laterality were examined. Based on teleost fish studies, we predicted that boldness would correlate with laterality, such that shyer individuals would be more strongly lateralized. Moreover, based on physiological evidence, we expected that right lateralized individuals (left eye use preference) would demonstrate higher stress reactivity than left lateralized individuals

\section{Materials and methods}

\subsection{Subjects}

Port Jackson sharks (Heterodontus portusjacksoni) were selected as study subjects due to their abundance in New South Wales coastal waters, as well as their small size and renowned hardiness, making them suitable for captive studies [43, 45]. A total of 17 juvenile $H$. portusjacksoni were opportunistically collected from three sites in New South Wales, Australia: 1) Middle Head, Mosman, NSW (3349’26.903”S $\left.151^{\circ} 15^{\prime} 50.404 ’ E\right)$; 2) Murray’s Sandline, Jervis Bay, NSW (354’23.015’S 150²7’28.188’E); 3) Broken Bay, NSW (3334’14.100’S 151¹7’15.200’E). Sharks from Middle Head and Murray’s Sandline were captured by hand, on snorkel, and sharks from Broken Bay were captured as by-catch on a commercial squid trawler. Sharks were 
housed at Sydney Institute of Marine Science (SIMS; Mosman, New South Wales) in four 1000-litre housing tanks (1.25 m diameter x $0.90 \mathrm{~m}$ depth). Sharks were separated into housing tanks according to total length; tanks 1 and 2 contained sharks $\leq 30 \mathrm{~cm}$ and tanks 3 and 4 contained sharks $>30 \mathrm{~cm}$. Tanks were supplied with fresh seawater from Chowder Bay, Mosman, NSW at ambient temperature, aerated, and furnished with approximately $5 \mathrm{~cm}$ of sandy substrate (taken from Chowder Bay) and PVC structures to provide shelter. The sharks were exposed to a natural light cycle.

For individual identification, sharks were tagged subcutaneously with an inert polymer elastomer (North-West Marine Technology Inc., Shaw Island, WA, USA) for and total length was measured. After 24 hours in captivity, sharks were fed to satiation with defrosted squid (Loligo opalescens). During non-experimentation periods, they were fed $\sim 5 \%$ body weight five days per week on a mixed diet of squid (L. opalescens and Nototodarus sloanii) and shrimp (Litopenaeus vannamei). Post experimentation, subjects were released at their original site of capture. Research was conducted under a scientific marine research ethics permit (ARA 2014/003) obtained from Macquarie University. All sharks were collected under NSW Department of Primary Industries permit P080010-4.2 and Australian Government permit AU-COM2014-259.

\subsection{Behavioural trait differences}

Sharks used in this study had individual differences in boldness and stress reactivity predetermined in a previous experiment [44]. Personality trials were conducted over a 17-day period, from April 23 to May 9, 2015. To prevent temporal autocorrelation, trials were separated by at least three days. Boldness trials were conducted for each shark 
once on days one, four, seven, and 14, and stress reactivity trials were conducted on days 10 and 17 of the testing period.

\subsubsection{Boldness}

The boldness assay described individuals' propensity to explore a novel habitat using an open-field emergence test, a classic test used to examine boldness in rodents [46] that has been successfully adapted for fish $[47,48]$. The testing arena consisted of a closed black Perspex refuge box $(0.75 \mathrm{~m} \times 0.75 \mathrm{~m} \times 0.25 \mathrm{~m})$ with a sliding door $(0.25 \mathrm{~m} \times 0.30$ m) leading into the open center of the experimentation tank (Intex Easy Set ${ }^{\circledR} 10$-foot pool; interior diameter: $2.3 \mathrm{~m}$ ) filled with seawater to $45 \mathrm{~cm}$ depth.

Subjects were placed into the starting compartment, where they were allowed to acclimate for two minutes. Immediately following acclimatization, the door was opened via a string pull and time until emergence was recorded. If sharks did not emerge after 15 minutes, the lid was removed to encourage sharks to leave the hide. If sharks failed to emerge after a total trial time of 20 minutes, the trial was terminated and sharks were given a maximum score of 1200 seconds [for further details see 44].

\subsubsection{Stress Reactivity}

Stress reactivity was examined utilizing changes in locomotory activity induced by acute handling stress. The testing arena consisted of a tank identical to housing tanks, and was supplied with a constant flow of fresh seawater from Chowder Bay at a rate similar to that of housing tanks. 
To induce stress, sharks were individually captured by hand and held out of water for one-minute. To minimize individual pre-test stress variability, sharks were only tested if successfully captured on the first attempt. Sharks were then placed into the testing tank, and the shark's behaviour was recorded for 30 minutes for each trial. Videos were later scored for activity levels via tail beat frequency. Tail beat frequency was counted by complete oscillations of the caudal fin within one-minute timeframes at predetermined times during the recording $[1,2,3,4,5,10,15,20,25$, and 30 minutes; 49]. Changes in activity were calculated by subtracting individual baseline activity rates from stressed activity levels [for further details see 44].

\subsection{Lateralization tests}

To ensure temporal separation from behavioural trait tests and separation of laterality tests, laterality in T-maze turn preferences and rotational swimming preferences were examined 14 and 30 days after the completion of personality trials, respectively.

\subsubsection{T-maze turn preference}

T-maze turn preferences were used to examine for laterality during exploration of a novel habitat. The apparatus consisted of a plus-maze that was transformed into a $\mathrm{T}$ maze by shifting a barrier to block the appropriate arm for each trial (Fig 1A). Testing consisted of 10 trials per individual.

Before each trial, sharks were allowed to swim freely throughout the entire maze and acclimatize for 15 minutes. Following this, the subject was confined to an enclosed starting compartment for two minutes while the unused arm was blocked off, bubblers 
were removed from inside the maze, and water was stirred. The front and top of the starting compartment were then removed, allowing the subject passage down the starting arm and into the maze. Sharks were given three minutes to leave the starting arm and turn either right or left into an adjacent arm of the maze. The first full 90-degree turn into an adjacent maze arm was recorded. If a shark did not select a turn direction within three minutes, the trial was terminated and excluded from analysis. Since $H$. portusjacksoni have little overlap in their visual fields, each eye effectively projects separately to the contralateral hemisphere [50-52]. Therefore, when turning right, $H$. portusjacksoni use their left eye to view the open, unexplored area of the maze. After a turn was made, sharks were completely enclosed in the end of the selected maze arm for a two-minute inter-trial interval while water was stirred and the barrier was moved to set up the maze for the following trial.

\subsubsection{Rotational swimming preference}

Rotational preferences have been used to measure laterality in several species of mammals [53-55] and fishes $[29,56,57]$. In this study we employed the same technique to describe motor laterality in $H$. portusjacksoni. To minimize stress during testing, experimentation occurred within housing tanks (1000 L aquaculture tubs; $1.25 \mathrm{~m}$ diameter x $0.90 \mathrm{~m}$ depth). To test sharks independently, all sharks were removed from their housing tank and placed in a holding tank identical to their housing tank. Then one shark was randomly captured from the holding tank and individually placed back into its housing tank for testing. One trial was conducted for each individual. 
Testing consisted of a 30-minute acclimation period to allow sharks to return to normal activity, followed by a 5-minute testing period, during which all swimming behaviours were recorded. Trials were video recorded via a GoPro Hero 3 Black Edition (GoPro, Inc., San Mateo, CA, USA) mounted above the center of the tank. Lateral asymmetries were analyzed from video recordings by radially dividing the tank into eight equal sections and counting how many times an individual crossed into a new section and from what direction (clockwise or counterclockwise; Fig. 1B). To eliminate the possibility of water currents influencing swimming direction, water inflow was turned of for the duration of the trial. In between trials, water was refreshed at a rate of 20 litres $\min ^{-1}$ for 30 minutes.

\subsection{Data analysis}

\subsubsection{Behavioural traits}

Repeatability of personality traits were examined using linear mixed-effects models fit by REML estimation. For further details on analysis, see Byrnes and Brown [44].

\subsubsection{Lateralization}

T-maze turn and rotational swimming preference laterality were analyzed by calculating a 'Laterality index' (IL) for each individual, where $\mathrm{I}_{\mathrm{L}}=$ (number of right turns - number of left turns) / (total number of turns). For the rotational swimming preference, counterclockwise crossing of barriers were considered left turns and clockwise crossing of barriers were considered right turns. IL is a continuous value, ranging from +1 to -1 , in 
which positive scores indicate a preference for rightward turns and negative values indicate a preference for leftward turns. 'Laterality strength' (Ls) was calculated by taking the absolute value of IL. Individuals were considered lateralized if Ls was greater than or equal to 0.5 , indicating that $75 \%$ or more turns were to a single side $[5,56]$. Population level departures from random choice in laterality direction $(\mu=0)$ and overall non-lateralization were estimated using two-tailed and one-tailed Wilcoxon signed rank tests, respectively $[10,58,59]$. General linear models were used to examine the influence of sex, population, time in captivity, total length, boldness and stress reactivity on lateralization direction and strength. Models were fit using an information-theoretic approach following the guidelines of Burnham \& Anderson [60]. If multiple models competed for the best fit, model averaging was used to determine the relative importance of fixed effects. Due to housing conditions causing co-linearity between length, population, time in captivity and social group, only the most important of these fixed effects was included in the final model. Chi-squared with continuity correction (due to small sample sizes) was used to test for differences of overall lateralization between sexes. All statistical testing was conducted in R Studio (Version 0.98.1103, R Core Team 2015) with an alpha level of 0.05 used to interpret significance.

\section{Results}

\subsection{Behavioural traits}

All individuals completed the boldness and stress reactivity tasks. Individuals demonstrated significantly repeatable individual differences in boldness (open field emergence time) and stress reactivity (post-stress activity rate changes). Emergence times 
were repeatable across all trials $(\mathrm{t}=4.787, P<0.001)$. Mean individual emergence time ranged from 10.50 to 932.75 seconds. Change in post-stress activity rate was repeatable across the two trials $(\mathrm{t}=2.748, P=0.015)$. Mean individual activity level changes varied from -34.25 tail beats $\min ^{-1}$ to 90.05 tail beats $\min ^{-1}$. For detailed results, see Byrnes and Brown (In Review).

\subsection{Lateralization}

All best-fit models included lateralization direction or strength as the response variable and sex, population, boldness, and stress reactivity as covariates.

\subsubsection{T-maze turn preference}

All 17 individuals completed the T-maze turn preference task. H. portusjacksoni did not demonstrate population level lateralization in the T-maze task. Of the 17 sharks, five demonstrated lateralization in their turn preferences. Models revealed that direction and strength of lateralization did not differ by sex or population and was not related to either boldness or stress reactivity. Five out of nine females were lateralized (Fig. 2), but direction of lateralization varied by individual; four were left lateralized and one was right lateralized. Males, on the other hand, did not demonstrate a turn preference (Fig. 2).

\subsubsection{Rotational swimming preference}

Laterality scores could not be determined for five sharks since they failed to move during the testing period. Thus these five sharks were removed from analysis and rotational swimming analysis was conducted using the remaining 12 sharks (six male and 
six female). H. portusjacksoni demonstrated a tendency for lateralized rotational swimming preference $(\mathrm{V}=66, P=0.018$; Fig. 2), with nine out of twelve sharks demonstrating preferred rotational swimming directions, but direction varied by individual. Two females preferred swimming clockwise, two preferred swimming counterclockwise and two had no preference. Of the males, four preferred swimming clockwise, one preferred swimming counterclockwise and the remaining one demonstrated no preference. No significant correlations (sex, population, boldness or stress reactivity) were found with preferred swimming direction. The strength of lateralization significantly correlated with individual stress reactivity $(\mathrm{t}=-5.792, P<$ $0.001)$ and $\operatorname{sex}(\mathrm{t}=-3.253, P=0.014)$, but did not correlate with boldness or population. We found that individuals that were more reactive to stress had stronger laterality scores (Fig. 3B).

\subsection{Lateralization across contexts}

Significantly more $H$. portusjacksoni were lateralized in rotational swimming preference than in T-maze turn preference $(\mathrm{F}=8.009, P=0.001$; Fig. 2). The direction of laterality, however, did not differ between the two laterality tests. Spearman rank correlation yielded highly significant correlations in individual laterality direction $(\mathrm{S}=$ 44.320, $P<0.001)$ and strength $(\mathrm{S}=70.928, P=0.005)$ between T-maze turns and rotational swimming. Of the 12 sharks that complete both tests, females (four of six) were significantly more lateralized across both tests than males (zero of six; $\chi^{2}=3.375, P=$ $0.033)$. 


\section{Discussion}

The findings of this study are novel as they demonstrated the first account of behavioural lateralization in an elasmobranch species. Contrary to expectations, $H$. portusjacksoni did not demonstrate population level lateralization. Instead, sharks demonstrated inter-individual variation in preferences while turning in a T-maze or swimming direction in a circular enclosure. Additionally, we found that individual strength of lateralization during rotational swimming was linked with individual stress reactivity. Expression of lateralization differed between males and females, and was generally more apparent in rotational swimming than the T-maze turn task.

The finding of individuality in laterality has been common amongst numerous species of nonhuman animals. In a study examining lateralization of 16 species of fish, Bisazza et al. [61] demonstrated that direction of laterality is individually dependent in six species. Moreover, a degree of individual variation was apparent within the remaining 10 species that showed population laterality biases where a few fish exhibited a strong preference to turn the opposite direction of their respective population bias. Similar individual variation has been seen in other vertebrate species including reptiles [14], birds [4], and mammals [55]. In this study, sharks demonstrated inter-individual variation in preferences of turning/swimming direction in both tests. Furthermore, individual IL and Ls was consistent between laterality tasks, indicating that lateralization is highly individual in Port Jackson sharks. We caution that these conclusions should be further corroborated with larger sample sizes. In addition, laterality should be investigated in other elasmobranch species to understand if the lateralization trends observed here are consistent with other benthic and free-swimming species. 
It is proposed that variation in laterality is maintained through a link with other inter-individual behavioural differences [13]. While no relationship was found between boldness and laterality, the observations in the present study corroborate that interindividual laterality variation may be maintained through a correlation with corticosterone levels and stress responses. Previous research has demonstrated that a high level of corticosterone during embryonic development impairs the development of cerebral lateralization in domesticated chicks. Resultantly, once hatched, these nonlateralized individuals had chronically higher levels of corticosterone and were more reactive to stressors [34]. Similarly, nonlateralized dogs and humans demonstrated higher emotional reactivity to stressors $[33,62]$. In contrast, the observations in this study indicated that more strongly lateralized $H$. portusjacksoni were more reactive to stress. The paucity of research makes it difficult to draw general conclusions; however, the contrasting evidence suggests a context specific, and perhaps species' specific, relationship between stress responses and lateralization. In Barnard et al.'s [63] study with sheep, lateralization was related to stress reactivity when activity level was used as a indicator of stress; whereas the use of vocalizations as an indicator of stress demonstrated no such relationship. While little evidence exists for a relationship in teleost fishes, Dadda et al. [64] described partial emotional reactivity differences between lateralized and nonlateralized Girardinus falcatus in two of four stress tests. It is clear that the link remains equivocal in animals and warrants further investigation. We suggest future research take a comparative approach to examine any discrepancies between various stress indicators (i.e. stress hormone levels, respiratory rate, activity levels, etc.). 
The inter-individual variation in lateralization observed in this study may also be maintained through a lack of evolutionary selective pressures acting on lateralization in H. portusjacksoni. Several studies have demonstrated that fishes from high predation habitats or that shoal tend to show greater population level laterality, indicating that predation pressure and shoaling tendency play important roles in the evolution of laterality in fishes $[9,23,61,65]$. The ability to efficiently process information about predators, separately from information on conspecifics' movements, is likely essential to maintaining shoal cohesion while evading predators [61]. Little is known about species that predate on $H$. portusjacksoni, but mortality rates have been shown to be exceptionally low after birth, indicating that little predation occurs [66, 67]. Armed with dark, disruptive coloration and calcified dorsal spines, juveniles avoid detection from predators by resting on complex backgrounds in the interface between seagrass beds, whereas adults rest in caves or kelp beds, where large predators are unlikely to have feasible attack angles [43]. Furthermore, there is no record of $H$. portusjacksoni demonstrating shoaling behaviours. Therefore, given the lack of predation pressure or regular socially cohesive behaviours, it is unlikely that strong selective pressures would select for population level laterality in $H$. portusjacksoni; further explaining the lack of population level laterality observed in this study.

Alternatively, we cannot rule out the possibility that the lack of a population bias was an artifact of the experimental conditions, which did not include a biologically relevant stimulus during trials. Population laterality biases have often been observed in fishes when inspecting a predator or conspecific $[29,37]$. However, when fish are free swimming without the presence of a stimulus, laterality biases often disappear [29]. In 
theory, one reason for defined laterality in animals is to enhance the simultaneous processing of multiple stimuli $[15,68]$. Without the presence of a biologically relevant stimulus, such as a predator or possible mate, test subjects may not have experienced substantial cognitive loading that requires partitioning of the processing of stimuli to different brain hemispheres, resulting in an absence of observed laterality biases [10].

Interestingly, more sharks showed laterality for rotational swimming preferences (9 of 16) compared to T-maze turn preferences ( 5 of 16). This may be attributed to the influence familiarity with an environment may have on laterality expression. When presented with the detour test for the first time, convict cichlids (A. nigrofasciatus) did not demonstrate lateralization. However, after 24 hours of familiarization with the testing arena the same cichlids demonstrated significantly stronger lateralization [69]. Similarly, sharks in this study demonstrated greater lateralization when tested in the familiar environment of their housing tank, compared to the novel T-maze environment. When placed in a novel environment, it would be most advantageous for individuals to fully assess the area for shelter and foraging patches before establishing individual preferences. Therefore, it would make sense that laterality differences only arise in familiar environments when all options for shelter and foraging have been assessed and the most advantageous preference can be accurately chosen.

Despite our relatively small sample size, our data suggested that laterality is likely sex dependent in elasmobranchs. About $50 \%$ of females were lateralized across both the T-maze turn and rotational swimming preference tests, whereas no males were lateralized across both tests. More prominent lateralization in female $H$. portusjacksoni may be explained by differences in male and female social behaviours, as well as the cognitive 
advantages of cerebral lateralization. Female $H$. portusjacksoni demonstrate social refuging behaviours throughout the year, whereas male aggregations have only been observed during the mating season $[42,43]$. Social recognition has been shown to be strongly lateralized behaviour, and is demonstrated as important influence on maintaining social cohesiveness in fishes $[6,61,70]$. The effectiveness of these social refuging behaviours is likely enhanced by the cognitive advantages of stronger cerebral lateralization, and may be particularly important during mating season when males regularly harass females. More strongly lateralized individuals demonstrate more efficient processing of multiple stimuli, prospectively due to the ability to partition tasks to separate parts of the brain [68]. In goldbelly topminnows (G. holbrooki), this task partitioning enabled lateralized females to forage more efficiently while attending to harassing males [71]. During mating, male H. portusjacksoni secure themselves for copulation by biting onto a female's pectoral fin and may even wrestle her into submission $[72,73]$. These mating bouts are energetically taxing and can leave open wounds, subjecting females to infection [74]. Given the physically taxing nature of elasmobranch mating and the continuous foraging strategy of $H$. portusjacksoni, this enhanced ability to attend to harassing males while meeting daily energy intake needs may be an important adaptation to ensure mating season success in females.

It is possible that reproductive behaviour is lateralized in $H$. portusjacksoni, as reproductive asymmetries have been reported in other elasmobranch species. Ebert et al. [75] found that only the left ovary functions in three species of catsharks, and in reef mantas, over $95 \%$ of mating scars have been observed on females' left pectoral fin (personal communication; Guy Stevens). Similarly, a preference for males to approach 
from one side during mating has been reported in poeciliid fish [76]. If $H$. portusjacksoni demonstrate similar reproductive asymmetries, female lateralization would be beneficial in processing information on male mating behaviours and may even facilitate insemination. It is currently unknown if elasmobranch species exhibit asymmetrical reproductive behaviours, as observations of elasmobranch mating behaviours have been rare and side preferences seldom quantified. More observations of elasmobranch mating are needed to elucidate possible asymmetries and the potential advantages it may confer in elasmobranch species.

\section{Conclusions}

In conclusion, our findings clearly demonstrated individual and sex biased variation in laterality in $H$. portusjacksoni. This study is the first to demonstrate behavioural lateralization in an elasmobranch species. Given the wide specious taxa and the multitude of behavioural niches filled by elasmobranchs, additional research is warranted in a variety of other species. Future work should examine if laterality trends observed in this study are consistent in species with contrasting life-styles, such as freeswimming houndsharks or requiem sharks.

In support of previous hypotheses, we suggest that variation in lateralization is partially maintained due to a link with stress reactivity, and possibly other personality traits. Previous research examining the link between personality and laterality in fishes has provided a mixture of supporting and contrasting evidence $[9,23,24]$, and thus the question of how and why certain personality traits correlate with laterality remains open. Additionally, mechanisms that influence laterality in different testing apparatuses are 
highly equivocal. Therefore, further work on the influence of habituation is warranted, and we suggest future investigations employ a comparative approach.

\section{Acknowledgements}

The authors would like to thank members of the MEG and BEEF labs at Macquarie University for assistance with shark collection and husbandry. Thanks also go to Josh Aldridge for assistance with housing set up and maintenance, and Rolf Norrington for collection of sharks. This work was supported by the Department of Biological Sciences at Macquarie University. This paper is contribution number XXX from the Sydney Institute of Marine Science. 


\section{References}

[1] Rogers LJ, Andrew R. Comparative vertebrate lateralization: Cambridge University Press, 2002.

[2] Frasnelli E, Vallortigara G, Rogers LJ. Left-right asymmetries of behaviour and nervous system in invertebrates. Neuroscience \& Biobehavioral Reviews. 36 (2012) 1273-1291.

[3] Rogers LJ. Lateralization in vertebrates: its early evolution, general pattern, and development. Advances in the Study of Behavior. 31 (2002) 107-161.

[4] Magat M, Brown C. Laterality enhances cognition in Australian parrots.

Proceedings of the Royal Society of London B: Biological Sciences. 276 (2009) 41554162.

[5] Sovrano VA, Dadda M, Bisazza A. Lateralized fish perform better than nonlateralized fish in spatial reorientation tasks. Behavioural brain research. 163 (2005) 122-127.

[6] Bisazza A, Dadda M. Enhanced schooling performance in lateralized fishes. Proceedings of the Royal Society of London B: Biological Sciences. 272 (2005) 16771681.

[7] Ferrari MCO, McCormick MI, Allan BJM, Choi RB, Ramasamy RA, Chivers DP. The effects of background risk on behavioural lateralization in a coral reef fish. Functional Ecology. 29 (2015) 1553-1559.

[8] Stier AC, Geange SW, Bolker BM. Predator density and competition modify the benefits of group formation in a shoaling reef fish. Oikos. 122 (2013) 171-178. 
[9] Brown C, Gardner C, Braithwaite VA. Population variation in lateralized eye use in the poeciliid Brachyraphis episcopi. Proceedings of the Royal Society of London B: Biological Sciences. 271 (2004) S455-S457.

[10] Bisazza A, Facchin L, Pignatti R, Vallortigara G. Lateralization of detour behaviour in poeciliid fish: the effect of species, gender and sexual motivation. Behavioural brain research. 91 (1998) 157-164.

[11] Facchin L, Bisazza A, Vallortigara G. What causes lateralization of detour behavior in fish? Evidence for asymmetries in eye use. Behavioural brain research. 103 (1999) 229-234.

[12] Brown C, Magat M. The evolution of lateralized foot use in parrots: a phylogenetic approach. Behavioral Ecology. 22 (2011) 1201-1208.

[13] Reddon AR, Hurd PL. Aggression, sex and individual differences in cerebral lateralization in a cichlid fish. Biology Letters. 4 (2008) 338-340.

[14] Hews DK, Castellano M, Hara E. Aggression in females is also lateralized: lefteye bias during aggressive courtship rejection in lizards. Animal Behaviour. 68 (2004) 1201-1207.

[15] Bisazza A, Brown C. Lateralization of cognitive functions in fish. In: Brown C, Laland K, Krause J, editors. Fish Cognition and Behavior: Wiley-Blackwell (2011). p. 298-324.

[16] Robins A, Lippolis G, Bisazza A, Vallortigara G, Rogers LJ. Lateralized agonistic responses and hindlimb use in toads. Animal Behaviour. 56 (1998) 875-881.

[17] Deckel AW. Laterality of aggressive responses in Anolis. Journal of Experimental Zoology. 272 (1995) 194-200. 
[18] Vallortigara G, Cozzutti C, Tommasi L, Rogers LJ. How birds use their eyes: opposite left-right specialization for the lateral and frontal visual hemifield in the domestic chick. Current Biology. 11 (2001) 29-33.

[19] Casperd JM, Dunbar RIM. Asymmetries in the visual processing of emotional cues during agonistic interactions by gelada baboons. Behavioural Processes. 37 (1996) 57-65.

[20] Austin NP, Rogers LJ. Limb preferences and lateralization of aggression, reactivity and vigilance in feral horses, Equus caballus. Animal Behaviour. 83 (2012) 239-247.

[21] Benelli G, Donati E, Romano D, Stefanini C, Messing RH, Canale A. Lateralisation of aggressive displays in a tephritid fly. The Science of Nature. 102 (2015) 1-9. [22] Nepomnyashchikh VA, Izvekov EI. Variability of the behavioral laterality in Teleostei (Pisces). Journal of Ichthyology. 46 (2006) S235-S242.

[23] Irving E, Brown C. Examining the link between personality and laterality in a feral guppy Poecilia reticulata population. Journal of Fish Biology. 83 (2013) 311325.

[24] Brown C, Western J, Braithwaite VA. The influence of early experience on, and inheritance of, cerebral lateralization. Animal Behaviour. 74 (2007) 231-238.

[25] Brown C, Jones F, Braithwaite V. In situ examination of boldness-shyness traits in the tropical poeciliid, Brachyraphis episcopi. Animal Behaviour. 70 (2005) 10031009. 
[26] Archard GA, Braithwaite VA. Variation in aggressive behaviour in the poeciliid fish Brachyrhaphis episcopi: population and sex differences. Behavioural Processes. $86(2011) 52-57$.

[27] Archard GA, Braithwaite VA. Increased exposure to predators increases both exploration and activity level in Brachyrhaphis episcopi. Journal of Fish Biology. 78 (2011) 593-601.

[28] Koolhaas JM, Korte SM, De Boer SF, Van Der Vegt BJ, Van Reenen CG, Hopster H, et al. Coping styles in animals: current status in behavior and stress-physiology. Neuroscience \& Biobehavioral Reviews. 23 (1999) 925-935.

[29] Bisazza A, Vallortigara G. Rotational swimming preferences in mosquitofish: Evidence for brain lateralization? Physiology \& Behavior. 62 (1997) 1405-1407. [30] Henry JP. Psychological and physiological responses to stress: The right hemisphere and the hypothalamo-pituitary-adrenal axis, an inquiry into problems of human bonding. Integrative Physiological and Behavioral Science. 28 (1993) 369387.

[31] Spivak B, Segal M, Mester R, Weizman A. Lateral preference in post-traumatic stress disorder. Psychological Medicine. 28 (1998) 229-232.

[32] Gordon DJ, Rogers LJ. Cognitive bias, hand preference and welfare of common marmosets. Behavioural brain research. 287 (2015) 100-108.

[33] Branson NJ, Rogers LJ. Relationship between paw preference strength and noise phobia in Canis familiaris. Journal of Comparative Psychology. 120 (2006) 176. 
[34] Freire R, Van Dort S, Rogers LJ. Pre-and post-hatching effects of corticosterone treatment on behavior of the domestic chick. Hormones and Behavior. 49 (2006) 157-165.

[35] Vallortigara G, Bisazza A. How ancient is brain lateralization. In: Rogers LJ, Andrew RJ, editors. Comparative vertebrate lateralization, New York: Cambridge University Press (2002). p. 9-69.

[36] Bisazza A, de Santi A. Lateralization of aggression in fish. Behavioural brain research. 141 (2003) 131-136.

[37] De Santi A, Sovrano VA, Bisazza A, Vallortigara G. Mosquitofish display differential left-and right-eye use during mirror image scrutiny and predator inspection responses. Animal Behaviour. 61 (2001) 305-310.

[38] Dadda M, Domenichini A, Piffer L, Argenton F, Bisazza A. Early differences in epithalamic left-right asymmetry influence lateralization and personality of adult zebrafish. Behavioural brain research. 206 (2010) 208-215.

[39] Brown C, Braithwaite VA. Effects of predation pressure on the cognitive ability of the poeciliid Brachyraphis episcopi. Behavioral Ecology. 16 (2005) 482-487. [40] Fine ML, McElroy D, Rafi J, King CB, Loesser KE, Newton S. Lateralization of pectoral stridulation sound production in the channel catfish. Physiology \& Behavior. 60 (1996) 753-757.

[41] Davies DH, Lowson R, Burch SJ, Hanson JE. Evolutionary relationships of a "Primitive" shark (Heterodontus) assessed by micro-complement fixation of serum transferrin. Journal of Molecular Evolution. 25 (1987) 74-80. 
[42] Christie A. Account of a mass aggregation of port Jackson sharks ' Heterodontus portusjacksoni' at point Cooke Marine Sanctuary, Victoria, Australia. The Victorian Naturalist. 132 (2015) 108.

[43] Powter DM, Gladstone W. Habitat-Mediated Use of Space by Juvenile and Mating Adult Port Jackson Sharks, Heterodontus portusjacksoni, in Eastern Australia. Pacific Science. 63 (2009) 1-14.

[44] Byrnes E, Brown C. Individual personality differences in Port Jackson sharks (Heterodontus portusjacksoni). Journal of Fish Biology. (In Review).

[45] Powter DM, Gladstone W. Demographic analysis of the Port Jackson shark Heterodontus portusjacksoni in the coastal waters of eastern Australia. Marine and Freshwater Research. 59 (2008) 444-455.

[46] Walsh RN, Cummins RA. The open-field test: A critical review. Psychological Bulletin. 83 (1976) 482.

[47] Brown C, Burgess F, Braithwaite VA. Heritable and experiential effects on boldness in a tropical poeciliid. Behavioral Ecology and Sociobiology. 62 (2007) 237-243.

[48] Raoult V, Brown C, Zuberi A, Williamson JE. Blood cortisol concentrations predict boldness in juvenile mulloway (Argyosomus japonicus). Journal of Ethology. 30 (2012) 225-232.

[49] Hunter JR, Zweifel JR. Swimming speed, tail beat frequency, tail beat amplitude, and size in jack mackerel, trachurus-symmetricus, and other fishes. Fishery Bulletin of the National Oceanic and Atmospheric Administration. 69 (1971) 253. 
[50] McComb DM, Tricas TC, Kajiura SM. Enhanced visual fields in hammerhead sharks. The Journal of Experimental Biology. 212 (2009) 4010-4018.

[51] Luiten PGM. Two visual pathways to the telencephalon in the nurse shark (Ginglymostoma cirratum). I. Retinal projections. Journal of Comparative Neurology. 196 (1981) 531-538.

[52] Graeber RC, Ebbesson SO. Retinal projections in the lemon shark (Negaprion brevirostris). Brain, Behavior and Evolution. 5 (1972) 461-477.

[53] Sobel N, Supin AY, Myslobodsky MS. Rotational swimming tendencies in the dolphin (Tursiops truncatus). Behavioural brain research. 65 (1994) 41-45.

[54] Glick SD, Shapiro RM, Glick SD. Functional and neurochemical mechanisms of cerebral lateralization in rats. In: Glick SD, editor. Cerebral lateralization in nonhuman species, London: Academic Press Inc. (1985). p. 157-183.

[55] Westergaard GC, Suomi SJ. Lateral bias for rotational behavior in tufted capuchin monkeys (Cebus apella). Journal of Comparative Psychology. 110 (1996) 199.

[56] Bisazza A, Sovrano VA, Vallortigara G. Consistency among different tasks of left-right asymmetries in lines of fish originally selected for opposite direction of lateralization in a detour task. Neuropsychologia. 39 (2001) 1077-1085. [57] Nepomnyashchikh VA, Gremyatchikh VA. The relation between structure of trajectory and handedness of direction of locomotion on the Oreochromys mossambicus Peters (Cichlidae). Zhurnal obshchei biologii. 54 (1993) 619-629. [58] Bisazza A, Pignatti R, Vallortigara G. Laterality in detour behaviour: interspecific variation in poeciliid fish. Animal Behaviour. 54 (1997) 1273-1281. 
[59] Takeuchi Y, Hori M. Behavioural laterality in the shrimp-eating cichlid fish Neolamprologus fasciatus in Lake Tanganyika. Animal Behaviour. 75 (2008) 13591366.

[60] Burnham KP. Model selection and multimodel inference: a practical information-theoretical approach. Burnham KP, Anderson DR, editors. New York: Springer; (2002).

[61] Bisazza A, Cantalupo C, Capocchiano M, Vallortigara G. Population lateralisation and social behaviour: a study with 16 species of fish. Laterality: Asymmetries of Body, Brain and Cognition. 5 (2000) 269-284.

[62] Watson D, Clark LA, Tellegen A. Development and validation of brief measures of positive and negative affect: the PANAS scales. Journal of personality and social psychology. 54 (1988) 1063.

[63] Barnard S, Matthews L, Messori S, Podaliri-Vulpiani M, Ferri N. Laterality as an indicator of emotional stress in ewes and lambs during a separation test. Animal cognition. 19 (2016) 207-214.

[64] Dadda M, Zandonà E, Bisazza A. Emotional responsiveness in fish from lines artificially selected for a high or low degree of laterality. Physiology \& Behavior. 92 (2007) 764-772.

[65] Bibost A-L, Brown C. Laterality influences schooling position in rainbowfish, Melanotaenia spp. PLoS One. 8 (2013).

[66] Powter DM, Gladstone W. Embryonic mortality and predation on egg capsules of the Port Jackson shark Heterodontus portusjacksoni (Meyer). Journal of Fish Biology. 72 (2008) 573-584. 
[67] McLaughlin RH, O'Gower AK. Life history and underwater studies of a heterodont shark. Ecological Monographs. 41 (1971) 271-289.

[68] Rogers LJ, Zucca P, Vallortigara G. Advantages of having a lateralized brain. Proceedings of the Royal Society of London B: Biological Sciences. 271 (2004) S420S422.

[69] Reddon AR, Hurd PL. Individual differences in cerebral lateralization are associated with shy-bold variation in the convict cichlid. Animal Behaviour. 77 (2009) 189-193.

[70] Sovrano VA, Bisazza A, Vallortigara G. Lateralization of response to social stimuli in fishes: a comparison between different methods and species. Physiology \& Behavior. 74 (2001) 237-244.

[71] Dadda M, Bisazza A. Lateralized female topminnows can forage and attend to a harassing male simultaneously. Behavioral Ecology. 17 (2006) 358-363.

[72] Powter DM, Gladstone W. The reproductive biology and ecology of the Port Jackson shark Heterodontus portusjacksoni in the coastal waters of eastern Australia. Journal of Fish Biology. 72 (2008) 2615-2633.

[73] Tricas TC, Le Feuvre EM. Mating in the reef white-tip shark Triaenodon obesus. Marine Biology. 84 (1985) 233-237.

[74] Pratt Jr HL, Carrier JC. A review of elasmobranch reproductive behavior with a case study on the nurse shark, Ginglymostoma cirratum. Environmental Biology of Fishes. 60 (2001) 157-188. 
[75] Ebert DA, Compagno LJV, Cowley PD. Reproductive biology of catsharks

(Chondrichthyes: Scyliorhinidae) off the west coast of southern Africa. ICES Journal of Marine Science: Journal du Conseil. 63 (2006) 1053-1065.

[76] Aronson LR, Clark E. Evidences of ambidexterity and laterality in the sexual behavior of certain poeciliid fishes. The American Naturalist. 86 (1952) 161-171. 


\section{Figure legends}

Fig. 1. Top view of the experimental set-up for the (A) T-maze and (B) Rotational swimming tasks. SC: starting compartment. Radial division used to establish laterality in rotational swimming task shown by dotted lines.

Fig. 2. Proportion of females (F) and males (M) showing lateralization in the T-maze (left; $n=17$ ) and Rotational swimming (right; $n=12$ ) tasks. Significant difference in population laterality between tasks $(\mathrm{W}=168.500, P<0.001)$ indicated with asterisks.

Fig. 3. Relationship between Laterality strength (Ls) and Stress reactivity in both the (A) T-maze and (B) Rotational swimming tasks. Correlation coefficients and p-values are as follows: Maze $\left(\mathrm{R}^{2}=-0.068, P=0.829\right)$ and Rotational $\left(\mathrm{R}^{2}=0.568, P=0.003\right)$. 

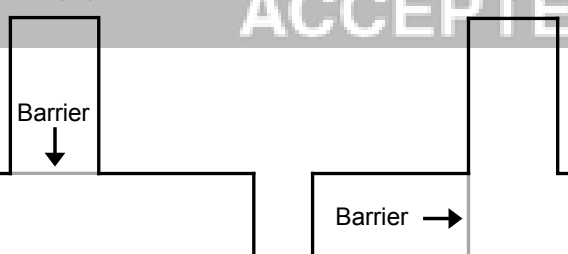

Water inlet
and outlet
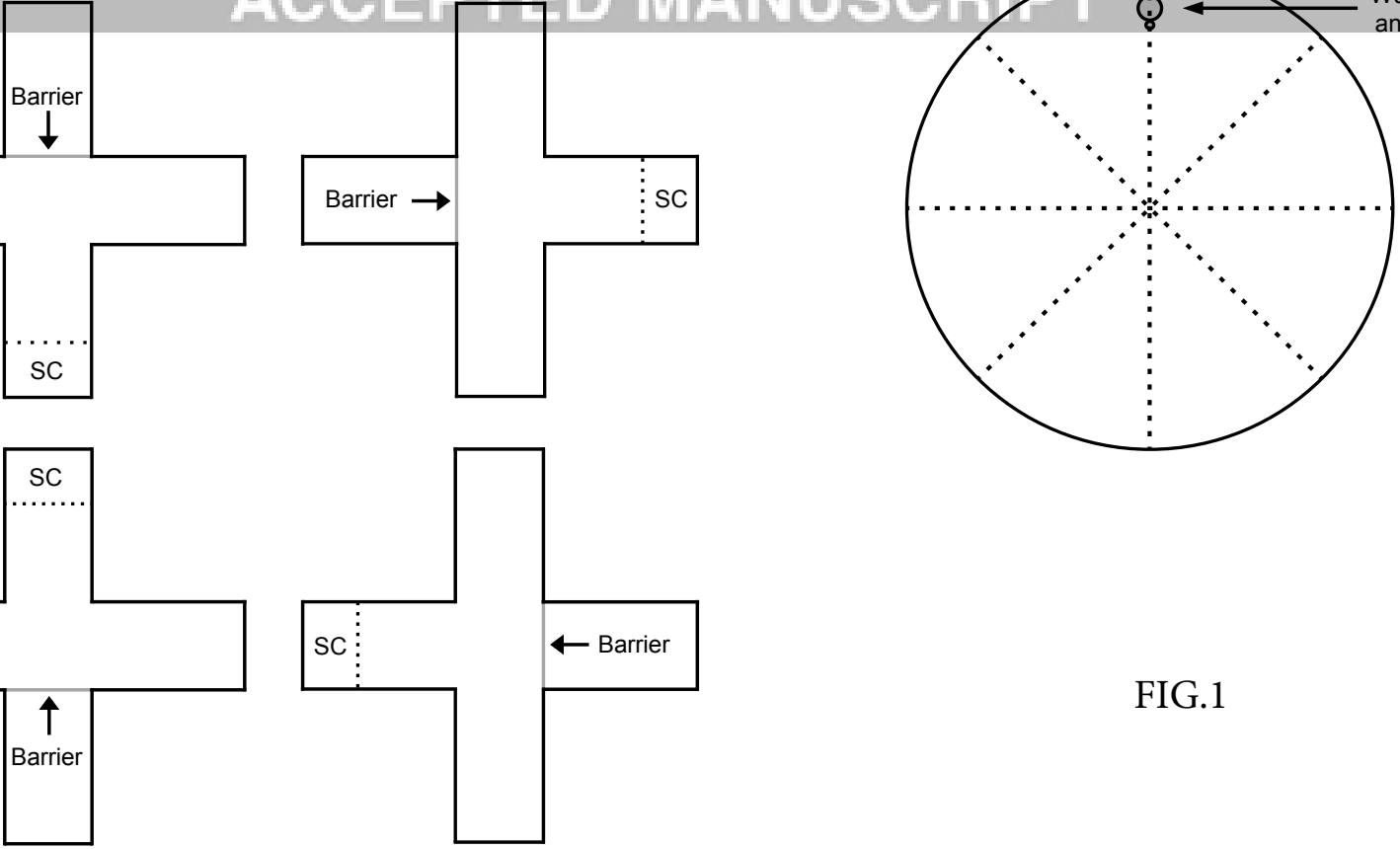

FIG.1 


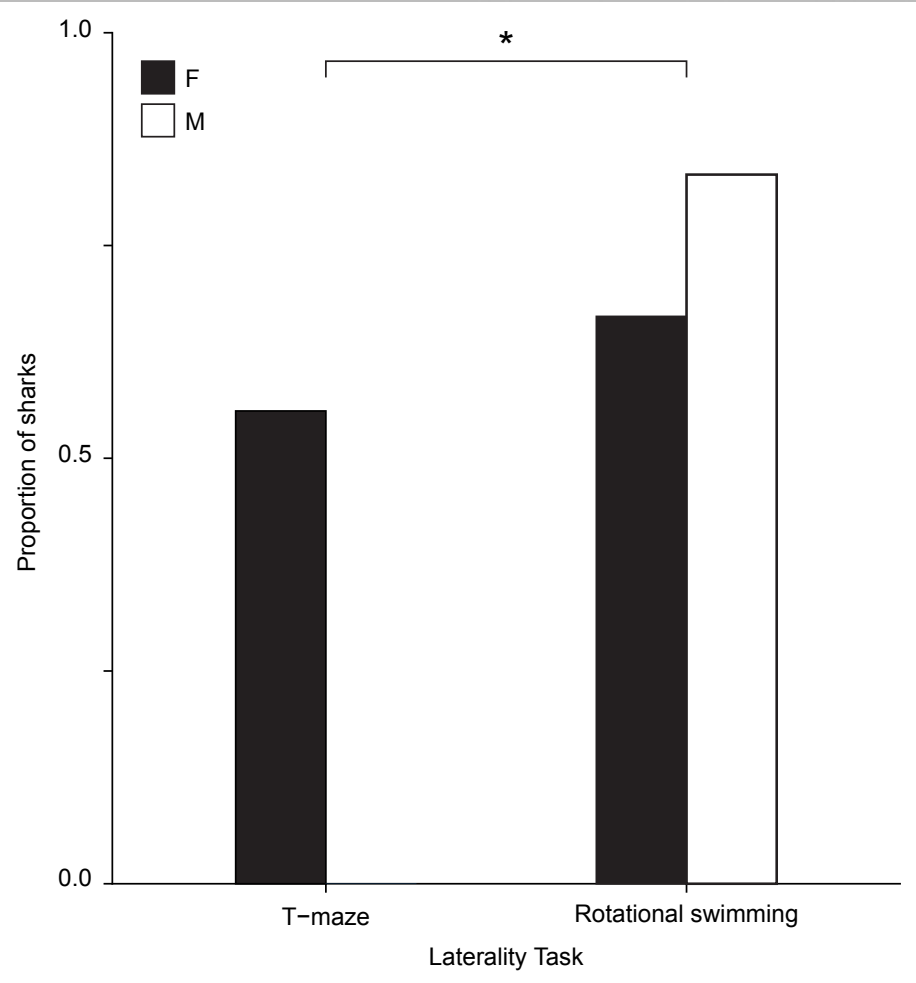

FIG.2 
